DETC2005-85242

\title{
MODELING AND EXPERIMENTAL DESIGN FOR THE ON-ORBIT INERTIAL PARAMETER IDENTIFICATION OF FREE-FLYING SPACE ROBOTS
}

\author{
Roberto Lampariello, Gerhard Hirzinger \\ German Aerospace Center (DLR) \\ Institute of Robotics and Mechatronics \\ 82230 Oberpfaffenhofen, Germany \\ Email: roberto.lampariello@dlr.de
}

\begin{abstract}
A method is proposed for the identification of the inertial parameters of a free-flying robot directly in orbit, using accelerometers. This can serve to improve the path planning and tracking capabilities of the robot, as well as its efficiency in energy consumption. The method is applied to the identification of the base body and of the load on the end-effector, giving emphasis to the experimental design. The problem of the identification of the full system is also addressed in its theoretical aspects.

The experience from the Getex Dynamic Motion experiments performed on the ETS-VII satellite have allowed to determine a most suitable model for the identification.
\end{abstract}

\section{Introduction}

Applications for free-flying robots in Earth orbit in the near future will involve complex tasks, such as maintenance and repair, on-orbit assembly, refueling and debris removal. These tasks will necessarily require a high system performance, in terms of reliability, efficiency and safety. Although teleoperation has been to date the only operational mode applied to such systems, shared and eventually full autonomy is a common development goal, as for example for the planned DLR technology demonstration experiment TECSAS (see Fig. 1).

This paper discusses the particular problem of identifying the inertial parameters, namely the mass, centre of mass position

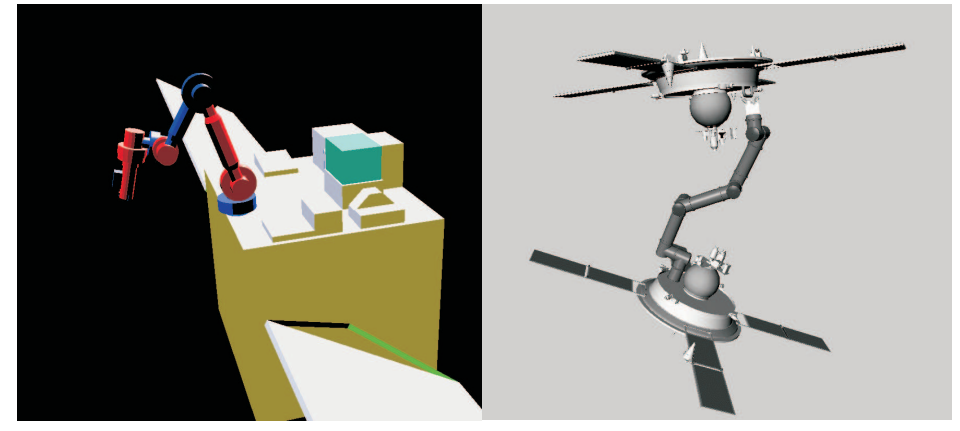

Figure 1. a. The ETS-VII free-flying robot; b. Simulation scenario for the TECSAS satellite servicing demonstration experiment

and inertia, of a free-flying robot and its load. The identification of the parameters of the base body (spacecraft) is first considered, where those of the remaining bodies, constituting the robot, are assumed given. Attention is then given to the opposite problem of identifying the inertial parameters of a load attached to the end-effector, where those of the base body and the robot arm are assumed known. Finally the problem of identifying the parameters of the whole system is addressed in its theoretical aspects.

The points which are addressed for the specified problems are as follows. Firstly, the experiment design for determining optimal exciting maneuvers for a free-floating robot is addressed, as this was pointed out in [1] as an open problem. Note that this 
is an important point because of the limited operational speed of the robot (for the ETS-VII experiments [2] a maximum endeffector velocity of $50 \mathrm{~mm} / \mathrm{sec}$. and $5 \mathrm{deg} . / \mathrm{sec}$., for the translational and the rotational components respectively, was allowed) and because of the particular base body dynamics.

Another open issue is the identifiability of the parameters, when those of the full system what to be identified. This problem reduces to determining the base inertial parameters for a freefloating robot.

A novel approach to the particular identification problem presented here, includes an analysis of the use of accelerometers to perform the identification, in terms of their applicability in orbit as well as their sensitivity requirements.

In this paper, only the inertial parameters defined above are considered for the identification problem. Joint dynamic parameters are conveniently eliminated by the use of rheonomically driven joints in the dynamic model. The inertial parameters of the robot arm can be assumed to be known from CAD data, generally to 5\% accuracy. Other parameters, such as those of the load, may have a large error (up to $100 \%$ or more). The method developed is applied in simulation to a three dimensional model with a six-degree-of-freedom manipulator and results are given based on such simulation and on the experience gained with the Getex experiments performed on the ETS-VII satellite in 1998 (see Fig. 1).

\subsection{Motivation for the identification of the inertial pa- rameters}

There are further considerations to make relative to the identification of the inertial parameters of free-flying robots. As already mentioned, robot motions in space are generally slow, due to restrictions on the base body reaction. As a first consequence of this, orbital disturbances become important in the correct description of the robot dynamics. Secondly, inertial parameter identification seems to be of little importance, since dynamic effects are small and should be easily dealt with by the controller. However, after noting the distinction between free-flying and free-floating robots in ones with actuated and non-actuated base body respectively, the two following points should be considered:

- the mapping between joint space and inertial (task) space of a free-floating robot is inertial parameter dependent;

- the aim of operational methodologies is to minimize the control effort of the base body, since fuel is in space a nonrenewable and expensive resource.

The following considerations then apply to the implementation of free-flying robot motion planning and control methodologies, in the presence of inertial parameter uncertainty.
1.1.1 Path planning issues Since the mapping between joint space and inertial space, where tasks are generally defined, is inertial parameter dependent, any path planning strategy which works in joint space, to the great advantage of avoiding dynamic singularities, but which is based on a desired final end-effector state and must satisfy given collision avoidance constraints, usually defined in inertial space [3], will derive erroneous solutions arising from the parameter uncertainty.

The only way to avoid this problem, is to implement a taskspace controller, i.e. to use sensor information from a reference in task space. The path tracker will then follow a path planning solution expressed in task space, diverging from its representation in joint space. This however will generally not be optimal for the given task and the true robot dynamics.

This argumentation comes to the conclusion that if path planning methods want to be used efficiently, inertial parameter identification has to be performed before any operational phase. If not, the solution will have to be tracked in task space and will not be optimal.

1.1.2 Control issues For the free-floating case, due to its nonholonomic nature, the control could be rather difficult to implement, if attention also needs to be given to the redundant degrees of freedom, in practice those of the base body, for purposes such as collision avoidance. In fact, the free-floating dynamics is such that the final state of the redundant degrees of freedom is path dependent. Therefore any undesired motion of the base would need to be corrected with complicated nonholonomic motions of the robot. The alternative to this problem is to actuate the base body, which again could result to be a suboptimal solution to the given problem.

Moving further into the control issue, let us assume that the suboptimal solution found by the path planner is acceptable for our purpose. The path tracker then has to follow the given solution in task space.

If adaptive control is implemented then the tracking error can be proven to be asymptotically stable but the parameter error will only be so in case persistent excitations are being performed by the robot (it is otherwise only bounded) [4].

This holds for the implementation of computed torque control with uncertainty and adaptation, in joint space. An adaptive control method has been developed for the free-floating case (although only 2D) in inertial space by $\mathrm{Gu}$ and $\mathrm{Xu}$ [5]. This can work correctly for tracking a desired path, but as explained above, this path will only be optimal if the inertial parameters are equal to those of the real system.

\subsection{Literature review}

The problem of parameter uncertainly or parameter drift has been tackled in the context of adaptive control by [5], [6] and [7]. The first addresses the problem of the identification of the param- 
eters of the robot-spacecraft system, in attitude controlled mode. The robot is modeled in joint space, for which it is shown that the unknown inertial parameters appear linearly. The authors, however, recognize the necessity to perform the identification before tracking any path in joint space and the need for persistently exciting inputs to determine the real parameter values.

The second [5] develops an "extended robot" model for the free-floating case, for which the inertial parameters also appear linearly. An adaptive control scheme is then developed based on the normal form augmentation approach but is applied to a twodimensional example only. Furthermore, the method relies, for the linear parameterization, on the measure of the base body position, velocity and acceleration, although it is not specified how this information is obtained. The third article [7] removes the necessity to measure the acceleration of the base body and suggests a two layer control system: one which carries out the identification (using the methods suggested by [5]); the other, on the base of the identification results, performs the tracking. The method is applied to a two-dimensional example, where reference is given to the use of exciting frequencies to determine persistently exciting inputs.

An off-line identification approach has also been developed in [1] and in [8], who apply the linear least squares identification method to the equations of a two body system (one joint), for which only the parameters of one are known. The identification problem is also addressed in [9], where that of the whole system is considered, with application to the realistic case of the ETSVII satellite (however only part of the parameters is identified, by only using the conservation of angular momentum). In none of the above examples is the problem of exciting maneuvers addressed in detail, especially not for the three-dimensional case, nor are the limitations of the identification of the whole system explained.

Parameter identification of ground robots has been extensively addressed, as for example by [10] and [11]. [10] develops a method based on the regressor matrix, minimizing its condition number to arrive at an optimal identification trajectory. No insight is given, however, on the design of the excitation maneuvers. In [12] it is argued that a correct treatment of noise is mandatory for efficient identification. An excitation-trajectory optimization method is therefore proposed, within a stochastic framework, which also minimizes the parameter uncertainty. However, in order to make use of an implementation in the frequency domain, the excitations are multi-periodic. This could give rise to the excitation of the flexible appendages and fluid elements of the free-floating robot and are as such not thought adequate.

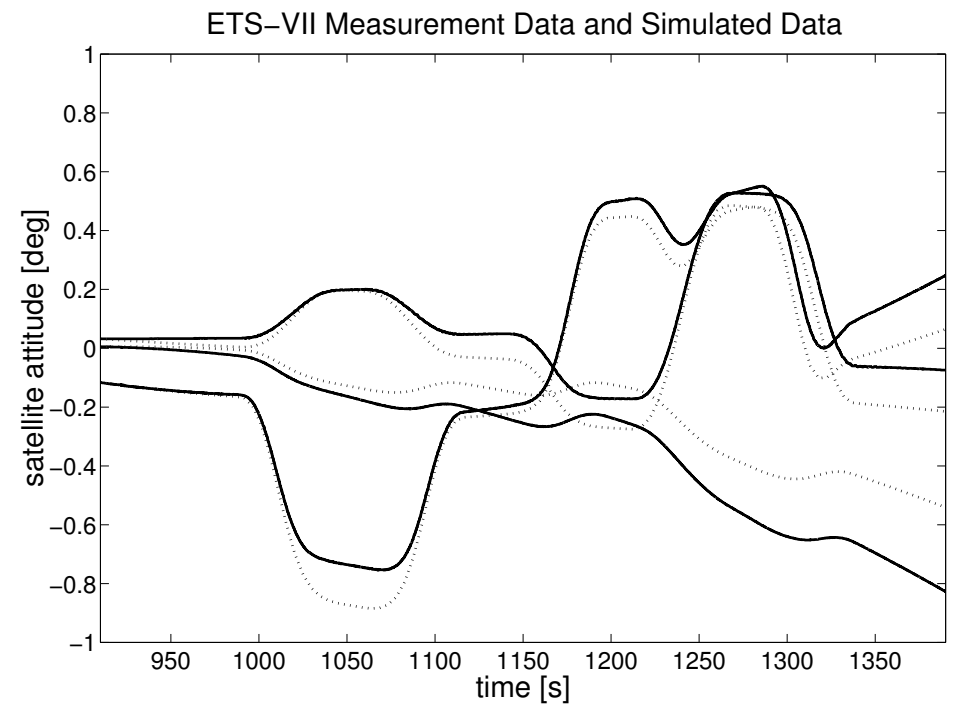

Figure 2. Measured (continuous line) and simulated (dotted line) satellite attitude (three Euler angles) for an experimental maneuver of the ETSVII satellite robot

\section{Modeling of the free-flying robot \\ 2.1 Orbital disturbances and the ETS-VII satellite ex- periments}

The Getex Dynamics Motion Experiments, performed by DLR in conjunction with NASDA on the Japanese ETS-VII experimental satellite, have outlined the importance of the disturbances in low Earth orbit on the motion of a free-floating robot. The experiments are reported in some detail in [2].

The main disturbances can be easily recognized when writing the equations of motion for the rotational dynamics of a gyrostatic body as follows [13]:

$$
\underline{I} \cdot \underline{\dot{\omega}}^{b I}=\underline{N}_{g g}-\underline{\omega}^{b I} \times\left(\underline{I}^{\prime} \cdot \underline{\omega}^{b I}\right)-\underline{\omega}^{b I} \times \underline{h}_{r w} .
$$

In this equation $\underline{\omega}^{b I}$ is the angular velocity of the satellite reference frame relative to the inertial frame on ground, $\underline{I}$ is the inertia tensor of the satellite with respect to its centre of mass and $\underline{h}_{r w}$ that of the reaction wheels with respect to the satellite. Also $\underline{N}_{g g}$ represents the gravity gradient torque.

From Eq. (1) it can be seen that the gravity gradient $\underline{N}_{g g}$, the residual angular momentum of the reaction wheels $\underline{h}_{r w}$ and the non-zero initial angular velocity of the satellite give rise to the drifting motion of the satellite found in the experimental results, shown in Fig. 2. The figure shows an example of the drift found in the experimental data, for a set of maneuvers performed on the free-flying robot while the attitude control of the spacecraft was switched off. Note that the robot motion was extremely limited, for operational safety and that the robot mass was a lot smaller 
than that of the satellite. Therefore, the disturbance of the base body motion was very small. The graph also indicates the fact that the simulated model gives rise to some discrepancy between the measured and the simulated data. This fact motivated the current work.

Furthermore, the assumption which is often made in deriving models for free-floating robots for simulation or control purposes, for which the system can be considered to be free of any external actions, is a rather strong one. This is even more so in the context of parameter identification, where modeling errors of such kind could be determinant. It is for this reason that the dynamical equations of motion, rather than their first integral representation, are used here for the identification.

\subsection{Equations of motion of a free-flying robot}

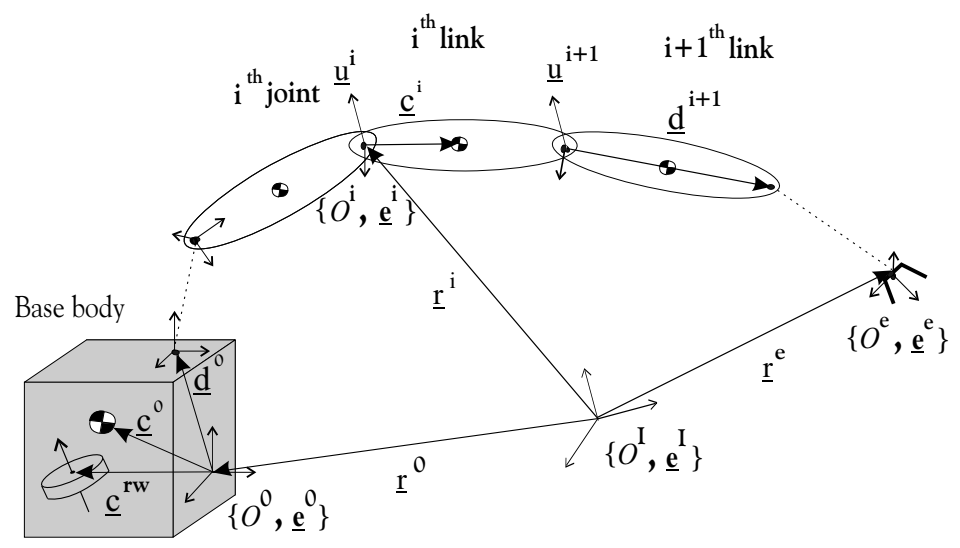

Figure 3. Reference frames and geometrical quantities of the multibody system

The equations of motion of a free-flying robot with rheonomically driven joints are now described (see [3] for more details). Consider the multibody system depicted in Fig. 3, composed by rigid bodies connected by revolute joints. The inertial frame of reference $\left\{O^{I}, \underline{\mathrm{e}}^{I}\right\}$ is initially taken to be in the Earth centre. This choice is dictated by the fact that the equations of motion are only valid when referenced to an inertial frame. Since the orbital frame is an accelerating frame, it usually cannot serve for this purpose.

Therefore, the quantity $\underline{r}^{i}$, shown in Fig. 3, refers to the orbital position vector of body $i$ and is clearly function of time. Also, the configuration of the $i^{t h}$ revolute joint, whose rotation vector is $\underline{u}^{i}$, is described by the variable $\theta^{i}$, which is measured relative to an arbitrary initial reference robot configuration.

The spatial equations of motion of the system can then be written, using the Newtonian-Eulerian formulation, as follows (kinematics are omitted for brevity, but details of the dynamic derivation will turn useful in the next section):

Dynamics:

$$
\begin{aligned}
& m^{i} \underline{\dot{v}}^{i}=\underline{f}_{e}^{i}+\underline{f}_{c}^{i} \\
& \underline{\underline{I}}^{i} \cdot \underline{\dot{\omega}}^{i}=\underline{t}_{e}^{i}+\underline{t}_{c}^{i}
\end{aligned}
$$

Constraints:

$$
\underline{r}^{i+1}=\underline{r}^{i}+\underline{d}^{i}
$$

$$
\mathrm{A}^{i+1}=\mathrm{B}^{i+1} \mathrm{~A}^{i}
$$

where $\underline{r}^{i}$ is the absolute position, $\mathrm{A}^{i}$ is the direction cosine matrix, $\underline{v}^{i}$ the translational velocity and $\underline{\omega}^{i}$ the angular velocity of body $i$ relative to the inertial frame. $\overline{\mathrm{B}}^{i}$ is the relative rotation matrix between frames $i$ and $i-1$, function of $\theta^{i}$. Furthermore, $m^{i}$ is the mass constant, $\underline{\underline{I}}^{i}$ the inertia tensor referred to the centre of mass, $\underline{f}_{e}^{i}$ and $\underline{t}_{e}^{i}$ are the sums of the external forces and torques and $\underline{f}_{c}^{i}$ and $\underline{t}_{c}^{i}$ are the sums of the constraint forces and torques (arising from the revolute joints) on the $i^{t h}$ body.

The equations of motion are resolved in the inertial frame $\left\{O^{I}, \underline{\mathrm{e}}^{I}\right\}$. Furthermore, the independent position state space variables are chosen to be

$$
\mathrm{y}_{I}=\left[\mathrm{r}^{0}, \phi^{0}, \theta\right]^{T} \mathrm{y}_{I I}=\left[\mathrm{v}^{0}, \omega^{0}, \dot{\theta}\right]^{T}
$$

where superscript 0 refers to base body quantities, $\phi^{0}$ is a column matrix $(3 \times 1)$ containing three attitude parameters (such as Cardan angles) and $\theta=\left[\theta^{i}\right]$.

The equations can be further modified to the advantage of the parameter identification, by expressing the joint motion as rheonomically driven, rather than acted upon by the motor torques. The result of this is that the equations are independent of any details of the modeling of the joints (in particular friction) since the rheonomic term itself expresses all these in terms of predefined motion variables $(\theta, \dot{\theta}$ and $\ddot{\theta})$. These can be obtained from measurement and as such be used for the identification of the inertial parameters which are of interest here.

It follows that the equations of motion become

$$
\mathrm{M} \dot{\bar{y}}_{I I}=\mathrm{C}+\Lambda^{0}+\chi
$$


where

$$
\overline{\mathrm{y}}_{I I}=\left[\mathrm{v}^{0}, \omega^{0}\right]^{T}
$$

In Eq. (7) $\chi$ is a linear function of $\ddot{\theta}$ and $\Lambda^{0}$ contains the sum of all external actions acting on the base body. Furthermore, matrix $\mathrm{M}$ and vector $\mathrm{C}$ represent the mass matrix and the apparent terms respectively.

\subsection{Orbital multibody dynamics model}

The equations of motion described in the previous section are only valid in an inertial frame of reference. It is evident though that the orbital frame is not inertial as it accelerates around Earth. In order for the equations of motion to be valid, they should be referenced to the Earth frame, which for our purposes could be assumed to be inertial. This is however impractical and it is necessary to eliminate the dependence of the equations on an accurate measurement of the orbital radius.

The left hand side of Eq. (2) is expressed using the relative derivative form (left superscript 'b') as follows:

$$
m^{i} \underline{\dot{v}}^{i}=m^{i}\left({ }^{b} \underline{\ddot{r}}^{i}+2 \underline{\omega}^{i} \times{ }^{b} \underline{\dot{r}}^{i}+\underline{\dot{\omega}}^{i} \times \underline{r}^{i}+\underline{\omega}^{i} \times \underline{\omega}^{i} \times \underline{r}^{i}\right) .
$$

Note that acceleration measurements of the spacecraft will consist of measures of $\underline{\ddot{r}}^{0}$, necessarily taken about its body frame. The centrifugal and the tangential terms on the right hand side of the equation shows that the measurement of vector $\underline{r}^{i}$ is in principle necessary.

The motion of the system can then be decomposed as follows (c.f. Eq. (2)):

$$
m^{i} \underline{\ddot{i}}^{i}=m^{i}\left(\underline{\ddot{R}}^{i}+\underline{\ddot{\varepsilon}}^{i}\right)=\underline{f}_{e}^{i}+\underline{f}_{c}^{i} .
$$

Vectors $\underline{R}^{i}$ and $\underline{\varepsilon}^{i}$ represent the reference orbital component and the additional component induced by the robot motion and orbital disturbances respectively.

An approach which is often used in the context of spaceflight dynamics, for the derivation of the relative motion between two spacecraft is that, for small orbital distances, the gravitational force can be expressed as a Taylor series expansions with respect to some reference point function [14]. Eq. (10) can first be expressed as

$$
m^{i}\left(\underline{\ddot{R}}^{i}+\underline{\ddot{\varepsilon}}^{i}\right)=\underline{g}^{i}(\underline{r})+\underline{f}_{c}^{i},
$$

where vector $\underline{g}^{i}$ is the gravitational force on body $i$, equal to $-m^{i}\left(\mu / r^{i 2}\right) \underline{\hat{r}}^{i}, r^{i}$ being the modulus of vector $\underline{r}^{i}$ and $\mu$ is the standard gravitational parameter of the Earth. When we expand this expression in a Taylor series about the point function for the base body, $-m^{0}\left(\mu / R^{02}\right) \underline{\hat{R}}^{0}$, we obtain, for the multibody system,

$$
m^{i}\left(\underline{\ddot{R}}^{0}+\underline{\ddot{\varepsilon}}^{i}\right)=\underline{g}^{i}\left(\underline{R}^{0}\right)+\underline{\underline{G}}^{i} \cdot \underline{\varepsilon}^{i}+\underline{f}_{c}^{i},
$$

having omitted all terms of order higher than $\underline{\varepsilon}^{2}$ and where $\underline{G}^{i}$ is the gravity gradient dyadic, given by the expression:

$$
\underline{\underline{G}}^{i}=\frac{\mu m^{i}}{R^{03}}\left[3 \hat{R}^{0} \hat{R}^{0}-\underline{\underline{u}}\right]
$$

$R^{0}$ is the modulus of vector $\underline{R}$ and $\underline{\underline{u}}$ is the unitary dyadic.

The first term on the right hand side of Eq. (12) can be eliminated together with the first term on the left hand side (note that this holds for any orbital eccentricity). The second term instead accounts for the distribution of the multibody system mass about the centre of mass of the base body in the gravitational field. Each $\underline{\varepsilon}^{i}$ can be expressed in function of $\underline{\varepsilon}^{0}$, relative to the first body, and of the geometrical quantities of the multibody system.

What is important to note is that the dependence of the equations on the orbital radius has now reduced to that in the gravity gradient dyadic expression, Eq. (13). However, a simple sensitivity analysis shows that an error in the measurement of $R^{0}$ of 1 $\mathrm{km}$ gives rise to an error in the torque of the order of $1 \mathrm{e}-6 \mathrm{Nm}$. Current orbital position measurement technology can do much more than that, as for example the Global Positioning System, for which precision of up to $1 \mathrm{~m}$ can be achieved. The resultant error in the gravity gradient expression can then be neglected.

Finally, end equations are:

$$
\begin{gathered}
m^{i} \underline{\ddot{\varepsilon}}^{i}=\underline{\underline{G}}^{i} \cdot \underline{\varepsilon}^{i}+\underline{f}_{c}^{i} \\
\underline{\underline{I}}^{i} \cdot \underline{\dot{\omega}}^{i}=\underline{t}_{e}^{i}+\underline{t}_{g g}^{i}+\underline{t}_{c}^{i},
\end{gathered}
$$

where

$$
\underline{t}_{g g}^{i}=3 \frac{\mu}{R^{3}} \hat{R} \times \underline{\underline{I}}^{i} \cdot \hat{R} .
$$

The new state space variables can be taken to be

$$
\mathrm{y}_{I}=\left[\varepsilon^{0}, \phi^{0}, \theta\right]^{T} \mathrm{y}_{I I}=\left[\dot{\varepsilon}^{0}, \dot{\omega}^{0}, \dot{\theta}\right]^{T}
$$


having expressed the vector quantities in the orbital frame. For the derivation of the equations of motion, Eq.s (14)- (15) then substitute Eq.s (2)- (3) in section 2.2.

The use of accelerometers is then possible and necessary for the measurement of the quantity $\dot{\varepsilon}^{0}$, as shown later.

\section{Statement of the identification problem and method of solution}

The parameter identification is separated into the following specific problems:

- problem 1: solve for the inertial parameters of the base body (or spacecraft);

- problem 2: solve for the inertial parameters of the load on the end-effector;

- problem 3: solve for the inertial parameters of the whole multibody system.

The excitation aims at maximizing the base body motion with suitable robot maneuvers and to using the measurements of the base body motion for identification.

The identification problem is written as a nonlinear leastsquares problem, based on an integral model of the equations of motion, as will be described shortly.

Each of the terms in the equations of motion (7) can be shown to be linear in the inertial parameters, or of linear combinations of them, more specifically:

$$
\mathrm{p}=\left[m^{i} m^{i} c^{i} \mathrm{I}^{i}\right]^{T}, \quad 1 \leq i \leq n,
$$

for $n$ bodies.

This leads to the possibility to express the equations in the form

$$
\Phi\left(\overline{\mathrm{y}}_{I}, \overline{\mathrm{y}}_{I I}, \dot{\overline{\mathrm{y}}}_{I I}\right) \mathrm{p}=\Lambda^{0}
$$

where $\Phi$ is generally termed the regressor matrix and vector $\Lambda^{0}$ represents the external forces on the base body, defined in equation 7. This gives rise to a standard linear estimation problem, the solution of which is then represented by a pseudo-inverse of $\Phi$. Generally speaking, the methods derived for example by [10] can then be applied to reduce the condition number of the regressor matrix $\Phi$ as much as possible. Note, however, that the right hand side is (nearly) zero, therefore the problem is badly posed. In order to use this method, the omission of rheonomically driven joints would be necessary, reintroducing the joint torques and the friction parameters as unknowns into the problem.

An alternative method can be defined as follows, based on a least squares approach applied to the integral of the equations of motion:

$$
\min _{\mathrm{p}} V(\mathrm{p})
$$

where

$$
V(\mathrm{p})=\sum_{i=1}^{N}\left\|\overline{\mathrm{y}}_{I I}^{i}-\overline{\mathrm{y}}_{I I}^{i} \exp \right\|^{2},
$$

$$
\overline{\mathrm{y}}_{I I}=\int_{0}^{t_{f}} \mathrm{M}^{-1}(\mathrm{p})\left(\mathrm{C}(\mathrm{p})+\Lambda^{0}+\chi(\mathrm{p})\right) d t
$$

In Eq. (21) $N$ is the number of experimental data points, collected in vectors $\bar{y}_{\text {II exp }}^{i}$, while in Eq. (22) $t_{f}$ is a generic final time of the excitation maneuver. Clearly, parameter $\mathrm{p}$ will relate to the base body for problem 1 , to the load for problem 2 and to the whole system for problem 3 .

\subsection{Base inertial parameters of free-floating robots with revolute joints}

In the case of problem 3 , the vector of unknown parameters ideally contains the inertial parameters for all the bodies of the system, i.e. $\mathrm{p}_{3}=\left[\begin{array}{llllll}m^{1} & m^{1} \mathrm{r}^{1} & \mathrm{I}^{1} & m^{2} & m^{2} \mathrm{r}^{2} & \mathrm{I}^{2}\end{array}\right]^{T}$. These must be reduced to the base inertial parameters.

The base inertial parameters are important in the identification procedure when the method described by Eq. (19) is used. In fact, with these parameters the resultant regressor matrix is non-singular for any state of the system. This is because the base parameters are linearly independent and all of them contribute to the system dynamics.

A description of the base parameters for a free-floating robot with revolute joints, based on the rules provided in [15] for a fixed based robot, follows. Of the classical inertial parameters given in Eq. (18), some generally result to have no effect on the dynamic model, or result to be linearly dependent.

For the parameters which have no effect on the dynamic model, the condition is that the column of the regressor matrix $\Phi$ which multiplies the given parameter, contains all zeroes. The rules given by [15] for these parameters are here modified according to the free-floating case:

1. There are no parameters having no effect on the dynamic model, since generally all links are free to translate and rotate in inertial space, in all directions. Therefore all parameters contribute to the dynamics. This is proven by observing the regressor matrix deriving from the equations of motion of a free-flying robot which include the torques at the joints (i.e. skleronomically driven). 
Regarding the linearly dependent parameters, the result found by [15], by which a revolute joint reduces the number of independent parameters by three, also applies to the free-floating case, as it is based on link energy considerations, independently of the constraints on the base body of the robot. As a result:

2. Some parameters are linearly dependent, in the same way as for a fixed-based robot. A revolute joint reduces the number of independent parameters by three.

The two rules are described by an example of a two joint free-floating robot (no base body actuation). For such a system, the equations of motion can be written as

$$
\Phi_{f f} \mathrm{p}_{f f}=\Lambda_{\text {joint }}
$$

where $\mathrm{p}_{f f}=\left[m^{1} m^{1} \mathrm{r}^{1} \mathrm{I}^{1} m^{2} m^{2} \mathrm{r}^{2} \mathrm{I}^{2}\right]$ and $\Lambda_{\text {joint }}$ represents the torques applied at the joints. After a QR decomposition of the regressor matrix $\Phi_{f f}$, it follows that the number of independent parameters is $(30-6)=24$, what we call here $K_{1}$.

The resulting base inertial parameters can be written in a vector array, $\mathrm{K}_{B}$. These parameter can be identified as discussed in the previous section. Furthermore, following [15], an expression can be written relating the parameters $\mathrm{K}_{B}$ to the independent and the dependent parameters $\mathrm{K}_{1}$ and $\mathrm{K}_{2}$, as

$$
\mathrm{K}_{B}=\mathrm{K}_{1}+\beta \mathrm{K}_{2},
$$

where $\beta$ is a constant matrix. From the knowledge of $\mathrm{K}_{2}, \mathrm{~K}_{1}$ clearly follows after the identification of $\mathrm{K}_{B}$.

\subsection{Degree of excitation}

It is proposed here that the degree of excitation of a maneuver is related to the amount of base body motion. Robot maneuvers are hence sought which maximize the base body motion, within the allowed bounds. This approach can be justified with the following argumentation. The cost function for the identification problem is given by Eq. (21). The condition of extremum, which provides the optimal solution, is then given by the equation

$$
\frac{\partial V}{\partial p_{i}}=2 \sum_{i=1}^{N}\left(\overline{\mathrm{y}}_{I I}^{i}-\overline{\mathrm{y}}_{I I}^{i}{ }_{\exp }\right) \frac{\partial \overline{\mathrm{y}}_{I I}{ }^{i}}{\partial p_{i}}=0, \quad 1 \leq i \leq m
$$

where $m$ is the number of parameters to be identified. Its solution is $\overline{\mathrm{y}}_{I I}{ }^{i}=\overline{\mathrm{y}}_{I I}{ }^{i}$ exp, $1 \leq i \leq m$.

From this is follows that the single derivatives of the state vector $\bar{y}_{I I}$ with respect to the parameters are weights for the errors between the estimated and measured values. The greater these weights, the greater the accuracy in the solution for which the errors are zero. The derivatives, which are in effect sensitivities of the states with respect to the inertial parameters, need to be as large as possible.

Consider then a single mass $m$ being acted upon by a force $F$, whose equation of motion is simply $m \ddot{x}=F$. It follows that the sensitivity of the output variable $\ddot{x}$ is given by $\frac{d \ddot{x}}{d m}=-F / m^{2}$. It is then evident that a greater value of $F$ will give rise to a greater sensitivity, what is useful for the purpose of the identification. This argument also holds for the integral form of this simple differential equation and therefore illustrates a physical property of mechanical systems, which justifies our excitation strategy.

\subsection{Experimental procedure}

The method consists in an iteration of the following operations:

- planning of optimal maneuvers with a simulation model and with guessed (or updated) parameters;

- execution of the optimal maneuver on the real system, with data acquisition;

- identification with a simulation model with update of the parameters.

3.3.1 Exciting maneuvers The first part is intended for planning the maneuvers for optimal excitation of the base body. An initial guess is given for the inertial parameters which are to be identified. With this initial guess, the algorithm determines an optimal exciting maneuver. In order not to damage any components of the system, the initial guess should be made conservative, such that the motion which will be executed by the real system will not be excessive.

The cost function for this algorithm is defined as

$$
\begin{aligned}
& V(\mathrm{q})_{\text {excitation }}=\left\|\overline{\mathrm{y}}_{\text {Imax }}\right\|^{2} \\
& -\left(y_{I 1 \max ^{2}}{ }^{2} y_{I 2 \max ^{2}}{ }^{2}\right)-\left(y_{I 1 \max ^{2}}{ }^{2} y_{I 3 \max ^{2}}{ }^{2}\right) \\
& -\left(y_{I 2 \max }{ }^{2}-y_{I 3 \max ^{2}}{ }^{2}\right)-\left(y_{I 4 \max ^{2}}{ }^{2}-y_{I 5 \max ^{2}}{ }^{2}\right) \\
& -\left(y_{I 4 \max }{ }^{2}-y_{I 6 \max }{ }^{2}\right)-\left(y_{I 5 \max }{ }^{2}-y_{I 6 \max }{ }^{2}\right) \text {, }
\end{aligned}
$$

where the subscript max relates to the maximum absolute value of the individual components $y_{I i}$ of vector $\bar{y}_{I}(1 \leq i \leq 6)$ during the maneuver. The cost function is formulated in such a way that large differences between the states $y_{I i}$ penalize its absolute value. Furthermore, for optimal excitation, the function is maximised in function of parameters q, to be defined below.

The excitation function parameterization is perhaps a key element of the procedure and is derived here from physical intuition. In order to excite all degrees of freedom of the base body, the first three joints of the robot are used, which usually constitute its shoulder and as such determine its greatest displace- 
ments. Hence, each of the joint motions, parameterized in time, is defined by the following equation

$$
\theta^{i}(t)=q_{1}^{i}+q_{2}^{i} \cos \left(\omega_{e} t\right)
$$

where the two parameters $q_{1}^{i}$ and $q_{2}^{i}$ appear for the amplitude of the oscillation and the initial position at which the oscillation takes place. An extra spline connects the initial state of each joint to position $q_{1}^{i}$ and similarly for the inverse motion after the sinusoid. The frequency of the oscillation is dictated by $\omega_{e}$. The remaining joints should be positioned such as to have the robot in the extended position. As conclusion, $\mathrm{q}=\left[q^{1}{ }_{1} q^{1}{ }_{2} q^{2}{ }_{1} q^{2}{ }_{2} q^{3}{ }_{1} q^{3}{ }_{2}\right]^{T}$

3.3.2 Identification The found solution to the first part is then executed on the real system (here only simulated on a system with parameters corresponding to the sought ones) and data is recorded. The second part of the identification then, finds a better guess to the unknown parameters. The cost function is simply given by Eq. (21).

\section{Results}

The nonlinear least square problems defined by Eq.s (21) and (26) was solved as an optimization problem with the Sequential Quadratic Programming method. All quantities are expressed in SI units.

\subsection{Problem 1: base body parameter identification}

The ETS-VII inertial parameters were taken as a first example, for which the base body parameters are the following: $\quad m_{\text {base }}=2645.9, \quad c_{\text {base }}=[-0.532,-0.281,1.499]^{T}$, $I_{\text {base }}=[5665.5,54.2,145.1 ; \ldots, 3033.0,-123.2 ; \ldots, \ldots, 7067.2]$ To test the method the parameters were then set to some initial guess value, with an initial large error: $\quad m_{\text {base }}=1000.0, \quad c_{\text {base }}=[0.5,0.5,0.5]^{T}, \quad I_{\text {base }}=$ $[1000.0,10.0,10.0 ; \ldots, 1000.0,10.0 ; \ldots, \ldots, 1000.0]$.

The initial guess of the excitation algorithm was instead taken to be $\mathrm{q}=[-1.1,-1.1,-1.1,-1.1,-1.1,-1.1]^{T}$. Such choice can very well be dictated by inspection of the robot kinematics and in order to favor an extensive robot motion. The joint limits were set to $\pm \pi / 2 \mathrm{rad}$.

For the first part of the procedure, the found solution to the excitation problem was found to be: $\mathrm{q}=$ $[0.63,-1.87,-2.18,-0.15,5.3 e-2,-0.56]^{T}$. The simulated response for the latter set of parameters is shown in Fig. 4. Note that the orbital angular motion is not shown in the graphs since these are describing the response with respect to the orbital reference frame. The results show that all states of the base body are excited to an approximately equal degree.
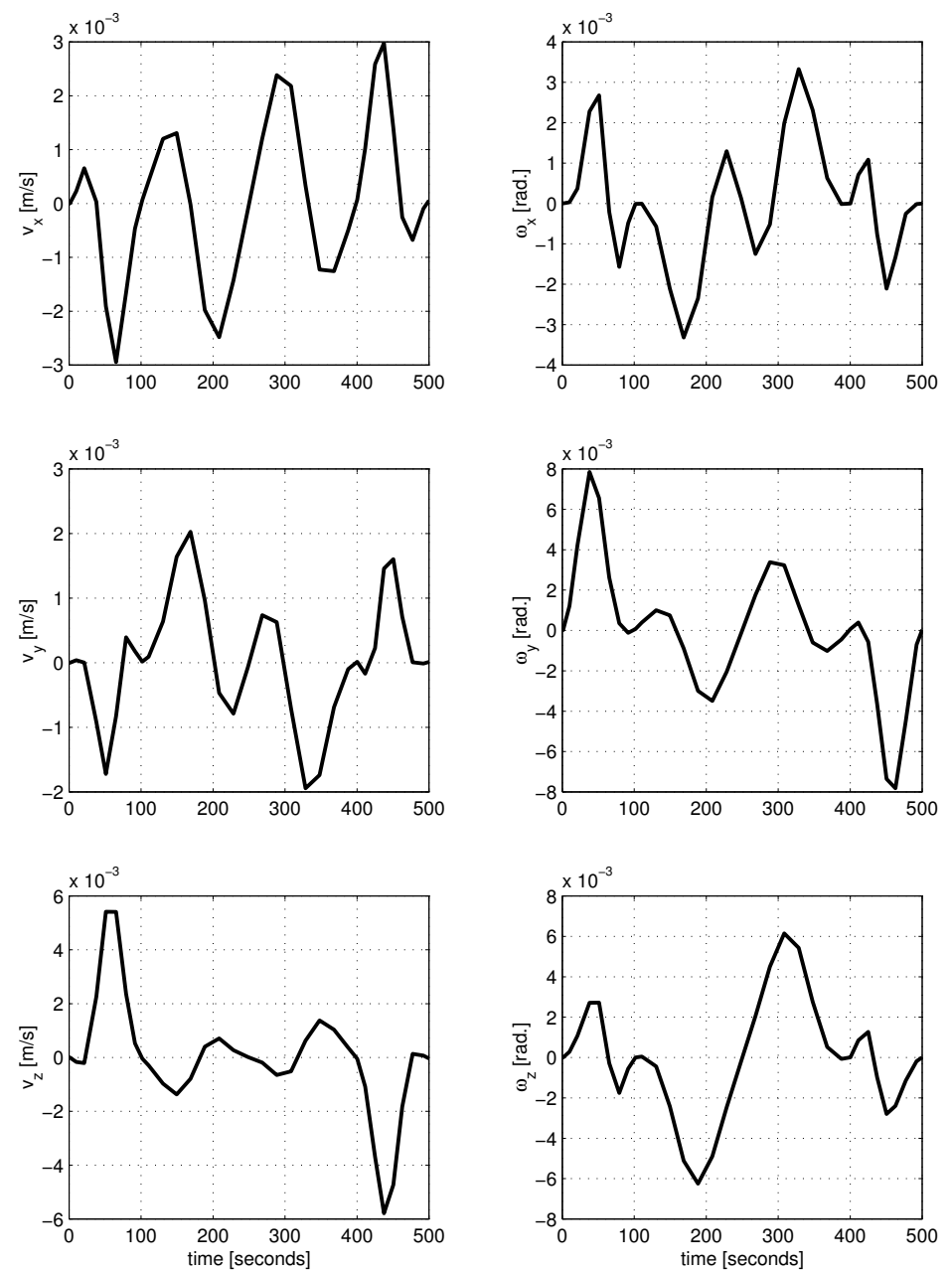

Figure 4. Base body state variables for excitation maneuver problem 1

The results of the second algorithm are then as follows: $m_{\text {base }}=2645.7, c_{\text {base }}=[-0.53,-0.28,1.50]^{T}, I_{\text {base }}=$ $[5667.25,50.98,148.39 ; \ldots, 3037.73,-124.13 ; \ldots, \ldots, 7075.85]$.

Following are the results for a poorly exciting maneuver, found with the following initial guess for the first algorithm: $\mathrm{q}=[0.5,0.5,0.5,0.5,0.5,0.5]^{T}$. The resulting solution of the first algorithm gives a corresponding base body motion for which the third rotational axis is excited by one order of magnitude less that the other two. The consequence of this is shown in the lost precision of the inertial estimate of the second algorithm: $m_{\text {base }}=2655.99, c_{\text {base }}=[-0.54,-0.23,1.53]^{T}, I_{\text {base }}=$ $[5539.09,42.70,161.246 ; \ldots, 3007.88,-57.47 ; \ldots, \ldots, 7070.54]$.

The inclusion of the gravity gradient into the equations of motion is found to be very important for the second algorithm, adding to the accuracy of the solution. Note that the value used for the orbital radius was taken as that of a Low Earth Orbit of altitude $538 \mathrm{Km}$, such that $r=6916 \mathrm{Km}$. 
The method was also found to adapt very well to systems with different mass distributions, for example such that the mass ration between the base body and the robot is increased to $1 / 7$, from $1 / 25$ of the ETS-VII.

\subsection{Problem 2: load parameter identification}

An example for the identification of the load parameters is given below. The load parameters are chosen arbitrarily to be: $m_{\text {load }}=370.0, \quad c_{\text {load }}=[0.33,0.50,1.04]^{T}$, $I_{\text {load }} \quad=\quad[700.0,0.0,0.0 ; \ldots, 700.0,0.0 ; \ldots, \ldots, 700.0]$. The initial guess was taken to be $\mathrm{p}=$ $[10.0,0.1,0.1,0.1,10.0,10.0,10.0,0.0001,0.0001,0.0001]^{T}$. After the first identification iteration, the results was found to be: $m_{\text {load }}=377.90, \quad c_{\text {load }}=[0.30,0.51,1.00]^{T}$, $I_{\text {load }} \quad=\quad[721.6,0.0,0.0 ; \ldots, 743.2,0.0 ; \ldots, \ldots, 710.9]$. After the second iteration, the solution was: $m_{\text {load }}=370.12, \quad c_{\text {load }}=[0.33,0.50,1.04]^{T}, \quad I_{\text {load }}=$ $[700.3,0.0,0.0 ; \ldots, 700.7,0.0 ; \ldots, \ldots, 700.2]$, which shows a clear improvement with respect to the result of the first iteration.

\subsection{Treatment of noise and sensor precision}

The inclusion of noise has already been treated in [16], where a noise with average standard deviation $\sigma=0.04$ was introduced in the simulated experimental data signals (note that the noise on the ETS-VII measurement data was found to be $\sigma=0.009$ ). An error on the resulting solution of $2 \%$ derived from this, after suitable treatment of the data (filtering and polynomial fitting). A similar error can be expected for the three dimensional case after the same procedure is adopted to the data. However, this has not yet been verified.

Furthermore, the joint measurements, which consist of position measurements, need to be differentiated twice to obtain their velocity and acceleration equivalents. This procedure can also be improved with filtering techniques [15] and with the use of position and torque sensors at the joints. However, the use of rheonomically driven joints eliminates the necessity for joint torque measurements, which are known to be much more noisy than position measurements [12]. The accelerometer signal must be integrated to obtain the required velocity input. The effect of this procedure was not yet analyzed.

The precision of the sensors is also very important for the results. The results given above have been performed with computer precision. Clearly this is only ideal. However, typical precision for rotational velocity measurements can be up to 2 arcsec [16], if a star sensor is available on board. Regarding translational acceleration, sensors are available for $10 e-5$ and up to $10 e-9 \mathrm{~ms}^{-2}$ precision, but perhaps only at very large costs.

\section{Conclusion}

Firstly, the paper shows the possibility to perform inertial parameter identification directly in orbit, by eliminating the need for inertial measurements from ground. This in principle allows the use of accelerometer measurements in the identification model. The necessity to perform identification of the inertial parameters has been motivated with a detailed analysis. The necessity to know part of the system parameters for identification of the whole system has also been described, by deriving the base inertial parameters of a free-flying robot. The choice of the dynamic model to perform the identification has been described in terms of realistic modeling necessity, deriving from the experience gained with the ETS-VII satellite experiments.

Secondly, a physically intuitive experimental design strategy was proposed, which is novel to the application in question. The excitation strategy is efficient and versatile for any load on the robot end-effector. The method has been applied to a threedimensional model within simulation and with insight gained from the past experimental experience.

\section{REFERENCES}

[1] Murotsu, Y., Tsujio, S., Senda, K., Ozaki, M.:Parameter Identification of Unknown Object Handled by Free-Flying Space Robot, Proc. of the 1992 AIAA Guidance Navigation and Control Conf., AIAA 92-4307, 1992.

[2] K. Landzettel, B. Brunner, G. Hirzinger, R. Lampariello, G. Schreiber, B.-M. Steinmetz: A Unified Ground Control and Programming Methodology for Space Robotics Applications - Demonstrations on ETS-VII, International Symposium on Robotics (ISR 2000), pp. 422-427, Montreal, Canada, May 2000.

[3] Lampariello, R., Agrawal, S., Hirzinger, G.: Optimal Motion Planning for Free-Flying Robots, IEEE International Conference on Robotics and Automation (ICRA 2003), Taiwan, 2003.

[4] Lewis, F.L., Abdallah, C.T., Dawson, D.M.: Control of Robot Manipulators, MacMillan Pulishing Company, New York, 1993.

[5] Gu, Y., Xu, Y.: A Normal Form Augmentation Approach to Adaptive Control of Space Robot Systems, Proceedings of the 1993 IEEE International Conference on Robotics and Automation, 1993.

[6] Xu, Y., Shum, H., Lee, J., Kanade, T.: Adaptive Control of Space Robot Systems with an Attitude Controlled Base, Space Robotics: Dynamics and Control, pp.229-268, Kluwer Academic Publishings, 1993.

[7] Shin, J., Lee, J.: Dynamics control with adaptive identification for free-flying space robots in joint space, Robotica, 12, pp. 541-551, 1994.

[8] Katoh, R., Sakon, H., Yamamoto, T.:A Control Method for Space Manipulator Mounted on a Fre-Flying Robot by Using 
Parameter Identification, Proceedings of the Asian-Pacific Vibration Conference '93, pp.1205-1209, 1993.

[9] Abiko, S., Yoshida, K.: Post flight analysis of ETS-VII space robotic experiments, $6^{\text {th }}$ International Symposium on Artificial Intelligence and Robotics and Automation in Space, St-Hubert, Quebec, Canada, 2001.

[10] Pfeiffer, F.: Parameter Identification for Industrial Robots, IEEE International Conference on Robotics and Automation, 1995.

[11] Armstrong, B.: On Finding Exciting Trajectories for Identification Experiments Involving Systems with Nonlinear Dynamics, The International Journal of Robotics Reserach, 8 , No. 6, Dec. 1989.

[12] Swevers, J. et al: Optimal Robot Excitation and Identification, IEEE Transactions on Robotics and Automation, 13, No. 5, October 1997.

[13] Wittenburg, J.: Dynamics of Systems of Rigid Bodies, B.G. Teubner Stuttgart, 1977.

[14] Clohessy, W. H., Wiltshire, R. S.: Terminal guidance system for satellite rendezvous, Space Flight Session, IAS National Summer Meeting, Los Angeles, June 16-19, 1959.

[15] Khalil, W., Dombre, E.,Modeling, Identification and Control of Robots, Hermes Penton Ltd, 2002.

[16] Lampariello, R., Hirzinger, G.: Freeflying Robots - Inertial Parameter Identification and Control Strategies, ESA Workshop on Advanced Space Technologies for Robotics and Automation (ASTRA 2000), ESTEC, Noordwijk, NL, December 2000 . 